\title{
A Crystalline Tri-thorium Cluster: When Metal o-Aromaticity Just Isn't Enough
}

\author{
Dariusz W. Szczepanik ${ }^{1,2^{*}}$
}

1. K. Guminski Department of Theoretical Chemistry, Faculty of Chemistry, Jagiellonian University, Gronostajowa, 2, 30-387 Kraków, Poland.

2. Institut de Quìmica Computacional i Catàlisi and Departament de Química, Universitat de Girona, C/ Maria Aurèlia Capmany, 69, 17003 Girona, Catalonia, Spain.

Corresponding author's email: dariusz.szczepanik@uj.edu.pl 
When Kekulé first pictured the ring structure of benzene, hardly anyone could have imagined that aromaticity may play a central role in an incredible variety of organic and inorganic rings containing $s^{-}, d$ - and even $f$-block metal atoms. ${ }^{1-4}$ Very recently, Liddle and coworkers synthesized the crystalline cluster $\left[\left\{\mathrm{Th}\left(\eta^{8}-\mathrm{C}_{8} \mathrm{H}_{8}\right)(\mu-\mathrm{Cl})_{2}\right\}_{3} \mathrm{~K}_{2}\right]\left(\mathbf{3}^{\prime \prime}\right)$ containing at its heart a threemembered ring built exclusively from thorium atoms. ${ }^{5}$ Remarkable stability and high symmetry of 3", the unexpected closed-shell ground-state electron configuration with a characteristic Highest Occupied Molecular Orbital (HOMO) reminiscent of three-center two-electron $\sigma$-bond, and the diamagnetic ring current induced under the external magnetic field - all these characteristics were deemed to be valid grounds for the compound to be considered $\sigma$-aromatic, thus extending the range of aromaticity to a record seventh row of the periodic table. However, Foroutan-Nejad et al. jumped in very promptly questioning $\sigma$-aromaticity of $\mathbf{3}$ " and demonstrating (using a different theoretical approach) that in the external magnetic field a weak paratropic ring current is induced around the tri-thorium core, providing a basis for the conclusion that 3 " is antiaromatic. ${ }^{6}$ Reconciling these contradictory predictions fits with the long-standing academic debate on the correspondence between aromatic stabilization (underlying symmetric structure and thermodynamic stability) and the magnetic-response properties of molecular rings. ${ }^{7}$

In the following, we provide multiple incontrovertible evidences that the crystalline cluster 3" is truly $\sigma$-aromatic, but the role of metal aromaticity is limited to shaping molecular-response properties rather than stability. An additional in-depth investigation of the resonance electronic structure of 3" reveals that the vital factor determining its high symmetry and thermodynamic stability is an unprecedented resonance bonding pattern involving thorium and chlorine atoms. 
To assess the effect of the $\sigma$-aromatic tri-thorium bonding on the stability of $\mathbf{3}$ " we designed its twin structure in silico with potassium atoms transmuted to argon atoms, thus depriving the original complex of two electrons delocalized between three thorium atoms. Consequently, in the resulting compound $\left[\left\{\mathrm{Th}\left(\eta^{8}-\mathrm{C}_{8} \mathrm{H}_{8}\right)(\mu-\mathrm{Cl})_{2}\right\}_{3} \mathrm{Ar}_{2}\right]\left(3^{*}\right)$ thorium atoms are no longer chemically bonded to each other, so the complex is clearly not $\sigma$-aromatic. But, to our great surprise, $3^{*}$ has the closed-shell ground-state structure that adopts the same symmetry and shares almost all the structural (Figure S1) and molecular-orbital characteristics of 3" (Figure S2), except noticeably longer distances between the thorium atoms and the much more distanced argon atoms. The infrared (IR) spectra of both compounds show a large overlap, and the corresponding molecular vibration contributions to the thermal energy differ by less than $1 \mathrm{kcal}$ mol $^{-1}$ (Figure 1a), suggesting rather marginal effect of the $\sigma$-aromatic tri-thorium bonding on the thermodynamic stability of 3". Furthermore, the relativistic energy gap between the HOMO and Lowest Unoccupied Molecular Orbital (LUMO) in $\mathbf{3}^{*}$ is found to be almost twice as large as the corresponding energy gap in 3" thereby marking the non-aromatic twin cluster even more stable (Figure S2). It should also be noted that although the HOMO in 3" is mainly made up of the $6 \mathrm{~d}$ thorium orbitals, the chlorine atoms contribute noticeably to its structure as well (Figure S3). The resulting inhomogeneity of the HOMO structure makes the magnetically-induced ring currents even more complex and difficult to assess as their character depends crucially upon the site of the thorium core (Figure S4a). This may explain the discrepancy between the predictions of (anti)aromaticity by Liddle and Foroutan-Nejad, ${ }^{5,6}$ since they were made using methodologies that differ dramatically in their sensitivity to non-local shielding effects from circulating electrons. $^{7}$ 


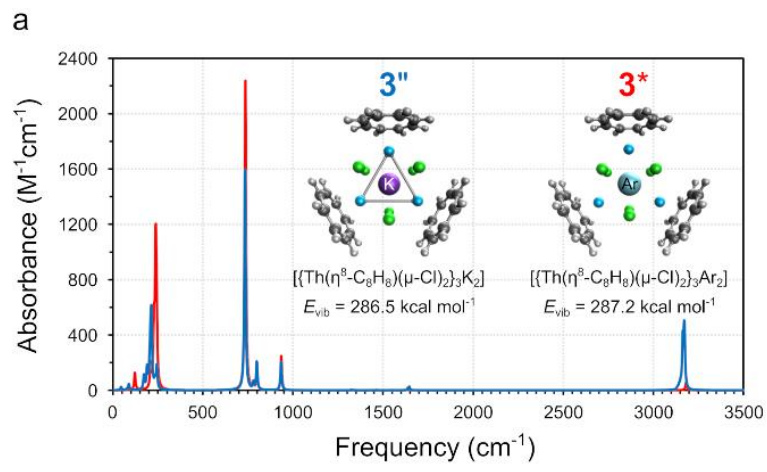

b

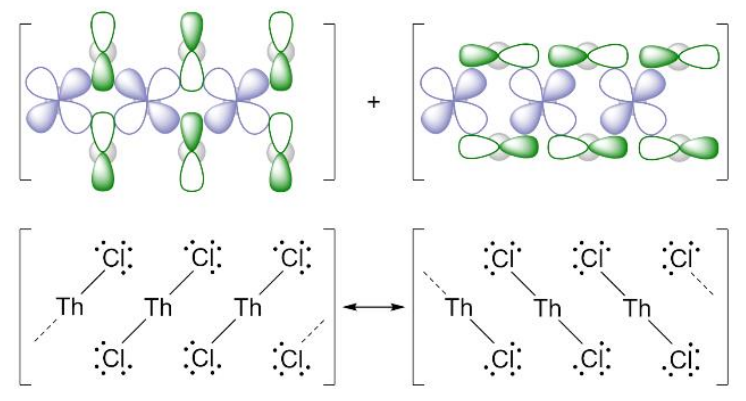

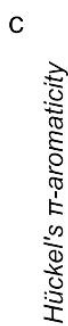
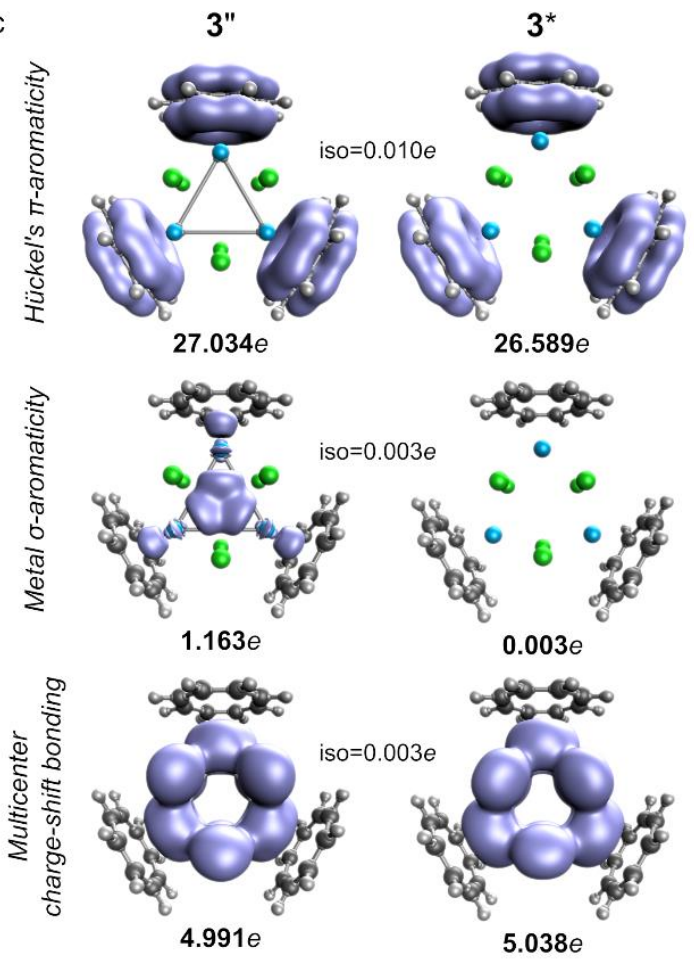

Figure 1. (a) The IR spectra of 3 " and $3^{*}$ with the corresponding molecular vibration contributions to the thermal energy. (2) Linearized side-view of two 6d-3p orbital conjugation topologies in which the in-phase overlapping of the thorium $6 \mathrm{~d}$ and the chlorine $3 \mathrm{p}$ orbitals is expected to be particularly effective; below, the associated resonance Lewis structures. (3) Isosurfaces (at given isovalues) of the EDDB function dissected into three contributions to the resonance stabilization; the corresponding bold numbers represent the populations of electrons 'resonating' between different bond positions.

Moreover, the highest occupied molecular orbitals in 3 " and $\mathbf{3}^{*}$ respond contrastingly to the external magnetic field (Figure S4), and only in the former a weak diamagnetic current density can be found around the centroid of the thorium core, marking its 'magnetic aromaticity'.

Having established that $\sigma$-aromaticity in the crystalline tri-thorium cluster determines the molecular-response properties but not the stability, the question remains: what kind of chemical interactions actually hold the entire $\mathrm{D}_{3}$-symmetric structure of $\mathbf{3}^{\text {" }}$ and $\mathbf{3}^{*}$ together? To answer that question, we need to take a cue from Figure $\mathbf{S 3}$ and turn our attention to the chlorine atoms surrounding the thorium ring. Analysis of possible conjugation topologies of the $6 \mathrm{~d}$ thorium and 
$3 p$ chlorine orbitals within the $\mathrm{Th}_{3} \mathrm{Cl}_{6}$ cage reveals two configurations in which the in-phase orbital overlapping is particularly effective (Figure 1b). The superposition of these orbital configurations enables a unique resonance mode with the polarized covalent bonds $\mathrm{Th}-\mathrm{Cl}$ and the corresponding charge-shift $\mathrm{Cl}$ :Th bonds ${ }^{8-10}$ cooperatively switching their positions (Figure 1b). Admittedly, each pair of thorium atoms does not sit in the same plane with the chlorine atoms, but due to extreme size of the $6 \mathrm{~d}$ orbitals (which easily penetrate the van der Waals spheres of chlorine atoms) the orbital overlapping is still expected to be very effective. The relativistic quantum-chemical calculations reveal the total valence ${ }^{11}$ of thorium and chlorine atoms to be equal 4.150 and 1.071 in 3 ", and 4.148 and 1.061 in $\mathbf{3}^{*}$, respectively. These values are close to the formal valency of thorium and chlorine atoms, IV and I, respectively, and the symmetry of the proposed resonance Lewis structures (Figure 1b) allows one to automatically predict the formal bond order between thorium and chlorine atoms to be equal 0.5 , which is completely in line with the calculated natural binding index of $0.462 .{ }^{12}$ Furthermore, a separate investigation shows that the geometric and electronic structure of the cyclooctatetraene (COT) ligands resembles to a large extent the Hückel's $10 \pi$-aromatic COT dianion (Figure S5) with two electrons promoted from each thorium atom. Putting these facts together gives a picture of bonding in the $\mathrm{Th}_{3} \mathrm{Cl}_{6}$ cage with thorium and chlorine atoms acting as divalent and monovalent elements, respectively, which perfectly correspond to the resonance Lewis structures representing the multicenter charge-shift bond (Figure 1b).

To quantitatively assess the magnitude of the multicenter charge-shift bonding in $\mathbf{3}^{\text {” }}$ and $\mathbf{3}^{*}$ the state-of-the-art theoretical approach called the Electron Density of Delocalized Bonds $(\mathrm{EDDB})^{10}$ was used. The EDDB method 'extracts' from the molecular wavefunction the information about electron pairs resonating between different bond positions, and thus it 
provides a unique tool to visualize and quantify any kind of the resonance-stabilized (or multicenter) bonding from typical $\pi$-aromatics to weak anagostic interactions. ${ }^{10}$ The results of the EDDB analysis (Figure 1c) show a clear and distinct picture of three types of the resonancestabilization effects in the tri-thorium complex: (1) Hückel's (4n+2) $10 \pi$-aromaticity of the COT dianionic ligands with the effectiveness of electron delocalization reaching $90 \%$ in $\mathbf{3}$ " (i.e., about $27.0 e$ out of the total $\pi$-population of $30.0 e$ contributes to resonance-stabilization); (2) the $\sigma$ aromatic three-thorium bond, in which only about $1.2 e$ can be associated with the stabilizing character; (3) the multicenter charge-shift bond in the $\mathrm{Th}_{3} \mathrm{Cl}_{6}$ cage with the total population of 5.0e effectively resonating over the thorium-chlorine bond positions. Therefore, the magnitude of resonance stabilization associated with the multicenter charge-shift bonding in $\mathbf{3}$ " is about four times greater than the corresponding effect from the tri-thorium $\sigma$-aromaticity. It is noteworthy that the average contribution of each chlorine atom to resonance stabilization in 3 " and $3^{*}$ (0.500e) is comparable to the corresponding effect in the dimer of aluminium chloride $(0.511 e)$, in which the charge-shift bonding is known to be strong enough to make $\mathrm{Al}_{2} \mathrm{Cl}_{6}$ stable in the liquid and even gas phase. ${ }^{14}$ Furthermore, the magnitude of the multicenter charge-shift bond remains unchanged even without the presence of $\sigma$-aromaticity in $3^{*}$, which perfectly corresponds to the structural and thermodynamic similarities between $\mathbf{3}^{\text {" and }} \mathbf{3}^{*}$, thus marking this newfound type of chemical bond as the actual cause of the uniqueness and beauty of the crystalline actinide cluster synthesized in the Liddle group. ${ }^{5}$

The results of this study prove fairly definitively that the $\sigma$-aromatic tri-thorium bond truly exists, but its principal role is limited to shaping molecular-response properties of $\mathbf{3}$ " rather than its stability. However, an additional in-depth investigation reveals that the thorium atoms put up a united front with the surrounding chlorine atoms to form an unprecedented multicenter charge- 
shift bonding pattern, which is demonstrated to be the primary factor underlying the high symmetry and remarkable stability of the tri-thorium cluster 3". The charge-shift bonds have recently been shown to be particularly strong when the $5 \mathrm{~d}$ orbitals of the transition-metals from group 11 and 12 are involved, ${ }^{15}$ but this study extends the range of the charge-shift bonding beyond transition metals to a high of principal quantum number six and to the seventh row of the periodic table.

The findings of this study have potential implications for future attempts to design and synthesize new actinide complexes by drawing attention to the essential stabilizing role of the actinide-halogen charge-shift bonding.

\section{Acknowledgments}

The author expresses his special thanks to Prof. Miquel Solà, Prof Stephen Liddle, and Dr. Josef T. Boronski for multiple stimulating discussions and critical reading of the manuscript. The research was partially supported by the European Union's Framework Programme for Research and Innovation Horizon 2020 (2014-2020) under the Marie Skłodowska-Curie, Grant Agreement No. 797335 (“MulArEffect”), and by the Polish National Agency for Academic Exchange under the Bekker programme, Grant Agreement No. PPN/BEK/2019/1/00219.

\section{Data availability}

Figures S1-5, XYZ coordinates, and details of the computational methods employed to perform the present work are presented in Supporting Information. 


\section{References}

1. Robinson, A. Chemistry's visual origins. Nature 465, 36 (2010).

2. Merino, G., Solà, M. Celebrating the 150th anniversary of the Kekulé benzene structure. Chem. Phys. Phys. Chem. 18, 11587-11588 (2016).

3. Zubarev, D.Y., Averkiev, B.B., Zhai, H.J., Wang, L.S., Boldyrev, A.I. Aromaticity and antiaromaticity in transition-metal systems. Phys. Chem. Chem. Phys. 10, 257-267 (2008).

4. Popov, I.A., Starikova, A.A., Steglenko, D.V., Boldyrev, A.I. Usefulness of the $\sigma$-Aromaticity and $\sigma$-Antiaromaticity Concepts for Clusters and Solid-State Compounds. Chem. Eur. J. 24, 292-305 (2017).

5. Boronski, J.T., Seed, J.A., Hunger, D., Woodward A.W., van Slageren J., Wooles A.J., L.S. Natrajan, Kaltsoyannis N., Liddle S.T. A crystalline tri-thorium cluster with $\sigma$-aromatic metalmetal bonding. Nature 598, 72-75 (2021).

6. Cuyacot, A.B.J., Foroutan-Nejad, C. Aromatic, or Antiaromatic, That Is the Question. ChemRxiv [Preprint] September 13, 2021 Version 1 (accessed September 15, 2021). Available from: https://doi.org/10.33774/chemrxiv-2021-3gv6v.

7. Zhao, L., Grande-Aztatzi, R., Foroutan-Nejad, C., Ugalde, J. M. \& Frenking, G. Aromaticity, the Hückel 4n+2 Rule and Magnetic Current. ChemistrySelect 2, 863-870 (2017).

8. Shaik, S., Danovich, D., Silvi, B., Lauvergnat, D.L., Hiberty, P.C., Charge-shift bonding - A class of electron-pair bonds that emerges from valence bond theory and is supported by the electron localization function approach, Chem. Eur. J. 11, 6358-6371 (2005).

9. Shaik, S., Danovich, D., Wu, W., Hiberty, P.C. Charge-shift bonding and its manifestations in chemistry. Nature Chem. 1, 443-449 (2009). 
10. Shaik, S., Danovich, D., Galbraith, J.M., Braïda, B., Wu, W., Hiberty, P.C. Charge-shift bonding: a new and unique form of bonding. Angew. Chem. Int. Ed. 59, 984-1001 (2020).

11. Szczepanik, D.W., Mrozek, J. Probing the interplay between multiplicity and ionicity of the chemical bond. J. Theor. Comput. Chem. 10, 471-482 (2011).

12. Glendening, E.D., Landis, C.R., Weinhold, F. NBO7.0: New vistas in localized and delocalized chemical bonding theory. J. Comput. Chem. 40, 2234-2241 (2019).

13. Szczepanik, D.W., Solà, M. The electron density of delocalized bonds (EDDB) as a measure of local and global aromaticity. "Aromaticity: Modern Computational Methods and Applications” (ed. I. Fernández), 259-283, Elsevier, 2021.

14. Helmboldt, O., Hudson, L.K., Misra, C., Wefers, K., Heck, W., Stark, H., Danner, M., Rösch, N. Aluminum Compounds, Inorganic. „Ullmann's Encyclopedia of Industrial Chemistry”, 2007.

15. Joy, J., Danovich, D., Kaupp, M., Shaik, S., Covalent vs Charge-Shift Nature of the MetalMetal Bond in Transition Metal Complexes: A Unified Understanding. J. Am. Chem. Soc. 142, 12277-12287 (2020). 